Draft Version OCtOBER 20, 2018

Preprint typeset using $\mathrm{IAT}_{\mathrm{E}} \mathrm{X}$ style emulateapj v. 5/2/11

\title{
BISPECTRUM OF THE SUNYAEV-ZEL'DOVICH EFFECT
}

\author{
Suman Bhattacharya ${ }^{1,2}$, Daisuke Nagai ${ }^{3,4}$, Laurie Shaw ${ }^{3,4}$, Tom Crawford ${ }^{5}$, Gilbert P. Holder ${ }^{6}$ \\ ${ }^{1}$ High Energy Physics Division, Argonne National Laboratory, Argonne, IL 60439 \\ 2 Kavli Institute for Cosmological Physics, The University of Chicago, Chicago, IL 60637 \\ ${ }^{3}$ Department of Physics, Yale University, New Haven, CT 06520 \\ 4 Yale Center for Astronomy \& Astrophysics, Yale University, New Haven, CT 06520 \\ 5 Department of Astronomy and Astrophysics, The University of Chicago, Chicago, IL 60637 and \\ ${ }^{6}$ Department of Physics, McGill University, Montreal, QC H3A 2T8, Canada \\ Draft version October 20, 2018
}

\begin{abstract}
We perform a detailed study of the bispectrum of the Sunyaev-Zel'dovich effect. Using an analytical model for the pressure profiles of the intracluster medium, we demonstrate the SZ bispectrum to be a sensitive probe of the amplitude of the matter power spectrum parameter $\sigma_{8}$. We find that the bispectrum amplitude scales as $B_{\mathrm{tSZ}} \propto \sigma_{8}^{11-12}$, compared to that of the power spectrum, which scales as $A_{\mathrm{tSZ}} \propto \sigma_{8}^{7-9}$. We show that the SZ bispectrum is principally sourced by massive clusters at redshifts around $z \sim 0.4$, which have been well-studied observationally. This is in contrast to the SZ power spectrum, which receives a significant contribution from less-well understood low-mass and highredshift groups and clusters. Therefore, the amplitude of the bispectrum at $\ell \sim 3000$ is less sensitive to astrophysical uncertainties than the SZ power spectrum. We show that current high resolution CMB experiments should be able to detect the SZ bispectrum amplitude with high significance, in part due to the low contamination from extra-galactic foregrounds. A combination of the SZ bispectrum and the power spectrum can sharpen the measurements of thermal and kinetic SZ components and help distinguish cosmological and astrophysical information from high-resolution CMB maps.
\end{abstract}

Subject headings: cosmology: dark matter — galaxies: clusters: general — intergalactic medium

\section{INTRODUCTION}

The Sunyaev-Zel'dovich (SZ) effect (Sunvaev \& Zeldovich 1980) is the principal source of CMB temperature anisotropy power at angular scales smaller than a few arcminutes. It comprises of two components, known as the 'thermal' and 'kinetic' effects. The former arises from inverse Compton scattering of CMB photons off hot electrons pervading galaxy cluster environments, distorting the Planckian form of the CMB spectrum. The kinetic SZ effect is caused by the Doppler shifting of CMB photons via Thomson scattering off clouds of electrons with a non-zero bulk velocity (along the line-of-sight) relative to the CMB rest frame. Recently, both the South Pole Telescope (SPT, Lueker et al. 2010; Shirokoff et al. 2011; Reichardt et al. 2011) and the Atacama Cosmology Telescope (ACT, Fowler et al. 2010; Dunkley et al. 2011) have placed constraints on the amplitudes of the kinetic and thermal SZ contributions to the small-scale CMB power spectrum. For instance, Reichardt et al. (2011) measured the thermal SZ amplitude at $150 \mathrm{Ghz}$ to be $3.69 \pm 0.65 \mu \mathrm{K}^{2}$ and placed an upper limit on the kSZ amplitude of $<2.8 \mu \mathrm{K}^{2}$ (at $95 \%$ confidence and assuming no SZ-point source correlation).

Measurements of the thermal SZ power spectrum probe the abundance-weighted pressure profiles of hot gas in groups and clusters over a wide range of redshifts. This is interesting in a cosmological context because the dependence on the abundance of clusters produces a sensitive scaling with $\sigma_{8}$; i.e., $C_{l} \propto \sigma_{8}^{(7-9)}$ (Komatsu \& Seliak 2002; Trac et al. 2011; Shaw et al. 2010, denoted as S10 hereafter). However, efforts to constrain $\sigma_{8}$ in this way have been inhibited by a large theoretical uncertainty on the amplitude of the tSZ signal (for a fixed cosmological model). Precise predictions of the SZ power spectrum have been hampered by the uncertainties in modeling the state of the intra-cluster medium (ICM) over a broad range of mass and redshift, and at large cluster-centric radii (Battaglia et al.|2010; Trac et al.|2011, S10). Early models and simulations produced predictions for the amplitude of the thermal SZ power spectrum that are discrepant with recent measurements by more than a factor of 2 (Lueker et al. 2010). Recent work, using hydrodynamical simulations (Battaglia et al. 2010, 2011), Nbody simulations +semi-analytic gas models (Trac et al. 2011) and analytic models (S10, Efstathiou \& Migliaccio 2011; Chaudhuri \& Majumdar 2011), have significantly reduced the tension between the observed and predicted values. However the distribution of amplitudes between different models and simulations is still significantly larger than the measurement errors (Reichardt et al. 2011), degrading the constraints that can be placed on cosmological parameters. Furthermore, as current experiments do not cover a sufficiently broad frequency range to enable the tSZ and kSZ signals to be distinguished spectrally, the kSZ contribution must currently be modeled and subtracted from the total SZ power, providing an additional source of systematic uncertainty.

In this work we propose the SZ bispectrum as a new, robust method for constraining the thermal SZ amplitude. The bispectrum, or its real-space counterpart, the 3 -point function, is widely used to measure the deviation of a signal from Gaussianity both in the CMB and in large scale structure data. The bispectrum in the CMB map can be measured by using three CMB tem- 
perature values and taking the average over the survey sky. The primordial CMB bispectrum can be used to search for signatures of primordial non-Gaussian fluctuations (see e.g., Komatsu et al. 2011, and references therein). The dominant secondary anisotropy signals in the CMB temperature power spectrum arise from lensing of the CMB by large-scale structure, the ISW effect and the SZ effect. These signals, either alone or from their cross correlation, give rise to a measurable bispectrum (Cooray 2000; Komatsu \& Spergel 2001; Cooray \& Hu 2000). The full non-Gaussian information (primordial or from low-redshift structure) can be obtained by Fourier transforming the CMB temperature field and taking the product of three temperature values that form a triangle, then summing over all possible triangle configurations in harmonic space, assuming all harmonic modes forming the triangles are independent.

One can also measure a skewness spectrum in which two sides of the triangle are summed over to measure the skewness as a function of the third side (Munshi \& Heavens 2010). The total skewness spectrum in a CMB map consists of that generated by CMB lensing, the thermal and the kinetic SZ effects, point sources, a signal arising due to the cross correlation of these components, and any primordial contribution. A significant fraction of the kinetic SZ power spectrum is generated by Gaussian density fluctuations in the linear regime (Shaw et al. 2011). Although patchy reionization may contribute a significant fraction of the $\mathrm{kSZ}$ spectrum (Mesinger et al. 2012), it is reasonable to expect that the kinetic SZ bispectrum is very small - the overall $\mathrm{kSZ}$ amplitude is 50-75\% smaller than the tSZ. The SZ skewness spectrum is thus likely to be dominated by the thermal SZ effect. As the primary CMB power decreases rapidly at $\ell>2000$, the CMB lensing bispectrum should also becomes negligible at high $\ell$. Cooray et al. (2000) have shown that the SZ skewness can be measured using the Planck data with a $\mathrm{S} / \mathrm{N} \approx 10$, assuming a perfect subtraction of the primary $\mathrm{CMB}$ and other foregrounds.

In this work, we focus on the SZ bispectrum, showing that it can provide information complementary to that from the SZ power spectrum. First, as the SZ skewness spectrum is dominated by the thermal SZ component it does not suffer from the observed thermal-kinetic SZ degeneracy. A combination of the SZ skewness and power spectra could therefore help disentangle the thermal and the kinetic signals. We show that the data from current small-scale CMB experiments have the potential to constrain the thermal SZ amplitude using bispectrum measurements with $5-10 \%$ accuracy. Furthermore, a tightened constraint on the kinetic SZ amplitude can also improve our understanding of the reionization scenario (Shaw et al. 2011; Zahn et al. 2011).

Second, as we will show, the signal is dominated by massive clusters at intermediate redshift for which highprecision X-ray observations exist. This is in contrast to the power spectrum where the signal mainly comes from the lower mass and higher redshift groups and clusters (e.g., Trac et al. 2011). The theoretical uncertainty in the SZ skewness spectrum is thus expected to be significantly smaller than that of the SZ power spectrum. Combined measurements of the power spectrum and the bispectrum can thus be used to distinguish the contribution to the power spectrum from different cluster mass and redshift ranges.

The goal of this study is to derive the SZ skewness spectrum using an analytic model for the ICM gas and analyze its astrophysics and cosmology dependence. In Section 2 we discuss the theory of the SZ bispectrum, and in Section 3 we describe our model for calculating the thermal pressure of the intra-cluster medium used in the bispectrum calculation. In Section 4 we present our results, including the distribution of the skewness spectrum signal over mass and redshift, the theoretical uncertainty, cosmological dependence, and the prospects of detectability from the current data from SPT like surveys. Section 5 presents summary and discussions. Throughout this work, we assume a fiducial cosmology with zero curvature and parameters: $\Omega_{b}=0.045, \Omega_{m}=$ $0.27, \sigma_{8}=0.8, h=0.71, n_{s}=0.97$, Komatsu et al. 2011) and $w_{0}=-1$.

\section{THEORETICAL FRAMEWORK}

\subsection{Sunyaev-Zel'dovich effect}

The Compton $y$-parameter for the thermal SZ effect is

$$
y=\frac{\Delta T}{T_{\mathrm{CMB}}}=\left(\frac{k_{B} \sigma_{T}}{m_{e} c^{2}}\right) \int n_{e}(l) T_{e}(l) d l,
$$

where $l$ is the distance along the line-of-sight; $n_{e}$ and $T_{e}$ are the electron number density and temperature, respectively.

The thermal SZ power spectrum can be calculated by summing up the square of Fourier transform of the projected SZ profile, weighted by the number density of clusters of a given mass and redshift,

$$
C_{l}=f\left(x_{\nu}\right)^{2} \int d z \frac{d V}{d z} \int d \ln M \frac{d n(M, z)}{d \ln M} \tilde{y}^{2}(M, z, \ell),
$$

where $V(z)$ is the comoving volume per steradian, $f\left(x_{\nu}\right)=x_{\nu}\left(\operatorname{coth}\left(x_{\nu} / 2\right)-4\right)$ with $x_{\nu}=h \nu /\left(k_{B} T_{\mathrm{CMB}}\right)$ at frequency $\nu$ and is negative (positive) below (above) $220 \mathrm{GHz}, T_{\mathrm{CMB}}$ is the temperature of CMB at $z=0$ and $n(M, z)$ is the halo mass function of Tinker et al. (2008), $\tilde{y}(M, z, \ell)$ is the Fourier transform of the projected SZ profile of a cluster, given by

$\tilde{y}(M, z, \ell)=\frac{4 \pi r_{s}}{\ell_{s}^{2}}\left(\frac{\sigma_{T}}{m_{e} c^{2}}\right) \int_{0}^{\infty} d x x^{2} P_{e}(M, z, x) \frac{\sin \left(\ell x / \ell_{s}\right)}{\ell x / \ell_{s}}$,

where $x=r / r_{s}, \ell_{s}=D_{A}(z) / r_{s}, r_{s}$ is the scale radius of the $3 \mathrm{D}$ pressure profile, $D_{A}(z)$ is the angular diameter distance at redshift $z$ and $P_{e}$ is the electron pressure profile. Note that we use the mass function fit for 400 times the mean density, whereas the pressure profiles are defined at the virial overdensity (Bryan \& Norman 1998). We convert the mass function definition to the virial density in Eq. 2. The cosmology dependence of the SZ power spectrum comes from the angular diameter distance and the growth function while the astrophysical dependence comes from the pressure profiles.

\subsection{Bispectrum Theory}

The CMB temperature fluctuations $\Delta \theta(\hat{\mathbf{n}})$ in a certain direction, $\hat{\mathbf{n}}$, can be expanded into spherical harmonics as

$$
a_{\ell m}=\int d^{2} \hat{\mathbf{n}} \frac{\Delta T}{T} Y_{l m}^{*}(\hat{\mathbf{n}}) .
$$


The angular bispectrum is

$$
B_{\ell_{1} \ell_{2} \ell_{3}}^{m_{1} m_{2} m_{3}}=\left\langle a_{l_{1} m_{1}} a_{l_{2} m_{2}} a_{l_{3} m_{3}}\right\rangle
$$

where the angle-averaged quantity in the full sky limit can be written as

$$
B\left(\ell_{1} \ell_{2} \ell_{3}\right)=\sum_{m_{1} m_{2} m_{3}}\left(\begin{array}{ccc}
\ell_{1} & \ell_{2} & \ell_{3} \\
m_{1} & m_{2} & m_{3}
\end{array}\right) B_{\ell_{1} \ell_{2} \ell_{3}}^{m_{1} m_{2} m_{3}}
$$

which has to satisfy the conditions: $m_{1}+m_{2}+m_{3}=0$, $\ell_{1}+\ell_{2}+\ell_{3}=$ even, and $\left|\ell_{i}-\ell_{j}\right| \leq \ell_{k} \leq \ell_{i}+\ell_{j}$. Here $B\left(\ell_{1} \ell_{2} \ell_{3}\right)$ is the bispectrum in the full-sky limit. In the small angle limit, the flat-sky approximation is valid. By defining $b\left(\ell_{1} \ell_{2} \ell_{3}\right)$ to be the bispectrum in the flat-sky limit, then the correspondence between the full-sky and the flat-sky bispectrum is ( $\mathrm{Hu}$ 2000),

$$
\begin{aligned}
B\left(\ell_{1} \ell_{2} \ell_{3}\right) & \left.\approx \sqrt{\frac{\left(2 \ell_{1}+1\right)\left(2 \ell_{2}+1\right)\left(2 \ell_{3}+1\right)}{4 \pi}}\left(\begin{array}{ccc}
\ell_{1} & \ell_{2} \ell_{3} \\
0 & 0 & 0
\end{array}\right) 7\right) \\
& \times b\left(\ell_{1} \ell_{2} \ell_{3}\right) .
\end{aligned}
$$

Throughout this work, we focus on the high- $\ell$ regime (to avoid confusion from primary $\mathrm{CMB}$ and its lensing), where a flat sky approximation is valid. We will refer to the flat-sky bispectrum; namely, $b\left(\ell_{1} \ell_{2} \ell_{3}\right)$ as the bispectrum for brevity and $B\left(\ell_{1} \ell_{2} \ell_{3}\right)$ as the full-sky bispectrum. Note that, for $\ell>500$, the Wigner 3 -j symbol can be approximated as

$$
\left(\begin{array}{ccc}
\ell_{1} & \ell_{2} & \ell_{3} \\
0 & 0 & 0
\end{array}\right) \approx \sqrt{\frac{2}{\pi}} \frac{(-1)^{L / 2}}{\left[L\left(L-2 \ell_{1}\right)\left(L-2 \ell_{2}\right)\left(L-2 \ell_{3}\right)\right]^{1 / 4}}
$$

if $L=\ell_{1}+\ell_{2}+\ell_{3}$ is even, and vanishes for odd $L$. The approximate expression for Eq. 8 is valid to better than 1 percent for $\ell>500$, the $\ell$ range we are interested in this study.

The thermal SZ bispectrum is the volume integral of the cube of the Fourier transform of the pressure profile weighted by the halo mass function,

$$
\begin{aligned}
b\left(\ell_{1} \ell_{2} \ell_{3}\right) & =f\left(x_{\nu}\right)^{3} \int d z \frac{d V}{d z} \int d \ln M \frac{d n(M, z)}{d \ln M} \\
& \times \tilde{y}\left(M, z, \ell_{1}\right) \tilde{y}\left(M, z, \ell_{2}\right) \tilde{y}\left(M, z, \ell_{3}\right) .
\end{aligned}
$$

We sum over the two smaller values of $\ell$ s and writing the bispectrum as a function of the largest $\ell$, we define the skewness spectrum as

$$
\Lambda(\ell)=\sqrt{\sum_{\ell_{1} \ell_{2}} b^{2}\left(\ell \ell_{1} \ell_{2}\right)}
$$

where $\ell+\ell_{1}+\ell_{2}$ is even and $\ell_{1} \leq \ell_{2} \leq \ell$.

In previous work, the amplitude of the thermal SZ power spectrum has been defined in terms of the expected amplitude at $\ell=3000$ for a given set of cosmological parameters (Lueker et al. 2010; Shirokoff et al. 2011; Reichardt et al. 2011; Dunkley et al. 2011). In this study, we define $A_{\mathrm{tSZ}}$ as the ratio of the thermal SZ power spectrum amplitude at non-fiducial cosmology to that at $\sigma_{8}=0.8$ as

$$
A_{\mathrm{tSZ}} \equiv \frac{C_{3000}\left(\sigma_{8}\right)}{C_{3000}\left(\sigma_{8}=0.8\right)}
$$

\begin{tabular}{|l|c|c|}
\hline \hline Parameters & Fiducial & Range \\
\hline \hline Dark Matter concentration $\left(A_{C}\right)$ & 1 & $0.8-1.2$ \\
Energy Feedback $\left(\epsilon_{f}\right)$ & $4 \times 10^{-7}$ & $(1-100) \times 10^{-7}$ \\
Dynamical Friction Heating $\left(\epsilon_{D M}\right)$ & 0.05 & $0.0-0.1$ \\
Non-thermal pressure normalization $\left(\alpha_{0}\right)$ & 0.2 & $0.0-0.3$ \\
Non-thermal pressure evolution $(\beta)$ & 0.5 & $-1-+1$ \\
\hline \hline
\end{tabular}

\section{TABLE 1}

Fiducial VAlues and the UnCERTAinty RANGe IN THE ICM PARAMETERS.

Similarly, we define the amplitude of the skewness spectrum as

$$
B_{\mathrm{tSZ}} \equiv \frac{\Lambda_{3000}\left(\sigma_{8}\right)}{\Lambda_{3000}\left(\sigma_{8}=0.8\right)}
$$

where $\Lambda_{3000}$ is the amplitude of the skewness spectrum at $\ell=3000$. For the fiducial cosmology, $B_{\mathrm{tSz}}=A_{\mathrm{tSZ}}=1$. Given that the skewness spectrum is proportional to the cube of the pressure profile the expected relation between $B_{\mathrm{tSZ}}$ and $A_{\mathrm{tSZ}}$ is $B_{\mathrm{tSZ}} \propto A_{\mathrm{tSZ}}^{1.5}$, assuming similar mass and redshift distribution of the power spectrum and the bispectrum. In Section 4.3 , we study the relation between the SZ amplitude of the power spectrum $\left(A_{\mathrm{tSZ}}\right)$ and the skewness spectrum $\left(B_{\mathrm{tsz}}\right)$ in more detail.

\section{MODELING THE INTRA-CLUSTER MEDIUM}

To calculate the thermal pressure profiles of groups and clusters, we use the physically motivated analytic model presented in S10. Here we briefly describe the key features of the model, and refer the readers to S10 for more details.

The model assumes that intra-cluster gas resides in hydrostatic equilibrium within the gravitational potential of the host dark matter halo,

$$
\frac{d P_{\mathrm{tot}}(r)}{d r}=-\rho_{g}(r) \frac{d \Phi(r)}{d r},
$$

where $\rho_{g}(r)$ is the gas density at radius $r$ from the cluster center, the total pressure, $P_{\text {tot }}(r)=P_{\mathrm{th}}(r)+P_{\mathrm{nt}}(r)$, is given by a sum of thermal and non-thermal pressures, and $\phi(r)$ is the gravitational potential. The gas is assumed to have a polytropic equation of state, $P_{\text {tot }}=$ $P_{0}\left(\rho_{g} / \rho_{0}\right)^{\Gamma}$, where $\Gamma=1.2$ and $P_{0}$ and $\rho_{0}$ are the central gas pressure and density respectively. The total mass distribution is assumed to follow the Navarro-Frenk-White (NFW) profile (Navarro et al. 1996, 1997),

$$
\rho_{\text {tot }}(r)=\frac{\rho_{s}}{x(1+x)^{2}},
$$

where $\rho_{s}$ is the normalization constant, $x=r / r_{s}$, and $r_{s}$ is the characteristic radius and can be defined in terms of the concentration of halos, $r_{s}=r_{\mathrm{vir}} / c_{\mathrm{vir}}$ where "vir" refers to the virial overdensity Bryan \& Norman 1998) with respect to the critical density of the universe. We adopt the concentration-mass relation given in Duffy et al. (2008):

$$
c_{\mathrm{vir}}(M, z)=7.85 A_{c}\left(\frac{M_{v i r}}{2 \times 10^{12} h^{-1} M_{\odot}}\right)^{-0.081}(1+z)^{-0.71},
$$




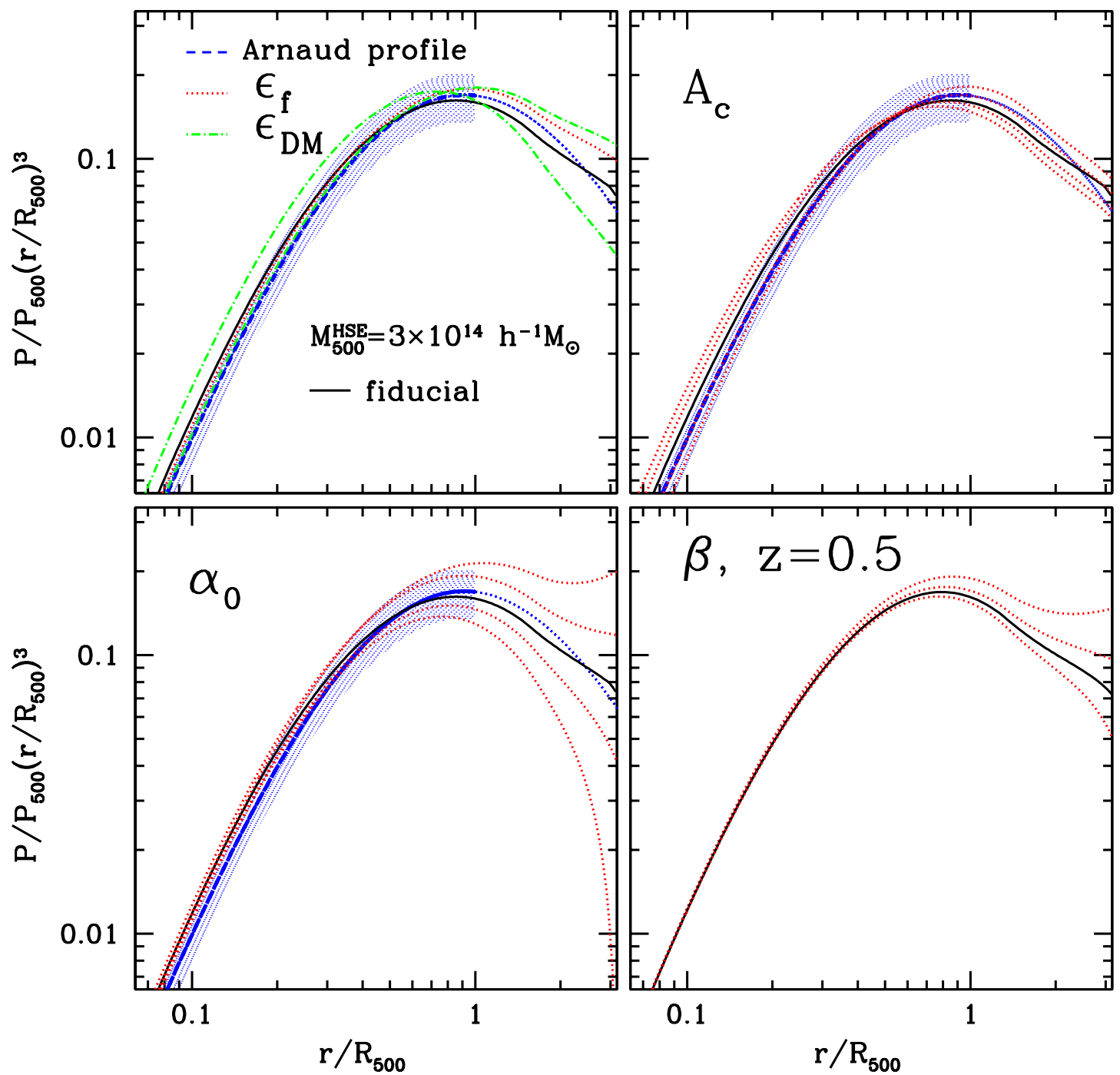

FIG. 1. - Comparison between the observed 'universal' pressure profile (scaled with respect to $P_{500}$ ) of (Arnaud et al. 2010) (blue dashed line, shaded region denotes $20 \%$ scatter) and our model predictions at $\mathrm{z}=0$ for a cluster of hydrostatic mass $M_{500}^{H S E}=3 \times 10^{14} h^{-1} M_{\odot}$. In each panel we vary one of the input model parameters to demonstrate their effect on the pressure profile. The black solid line represent the fiducial values as given in Table 1 The top-left panel shows the profiles obtained when $\epsilon_{f}$ is $10^{-5}$ (red dotted line) and $\epsilon_{D M}$ varies from 0.2 to 0.0 (green dashed lines, from top to bottom at $2 R_{500}$ ); in the top right panel we vary $A_{c}$ from $0.8,0.9,1.1,1.2$ (red dotted lines, top to bottom at $2 R_{500}$ ), the bottom-left panel shows the variation with $\alpha_{0}$ from $0.3,0.24,0.14,0.0$ (red dotted lines, bottom to top at $\left.2 R_{500}\right)$ and the bottom-right panel shows the variation with $\beta$ at $z=0.5$ over $\{1,0.5,0,-1\}$ where self-similar evolution is factored out (red dotted lines, from bottom to top). Note that the observed result is at $<R_{500}$, the blue dotted line is an extension of the Arnaud profile based on the simulation result.

where $A_{c}$ is the normalization factor, with the fiducial value $A_{c}=1$. Note that the gas is distributed as a massless tracer of dark matter distribution.

The model accounts for non-gravitational processes that affect the thermal properties of the ICM including star formation, energy feedback from supernovae (SNe) and Active Galactic Nuclei (AGN), and non-thermal pressure support due to bulk gas motions and turbulence. Star-formation is implemented by converting a certain (mass and redshift-dependent) fraction of the gas mass into stars. We adopt the observed stellar mass fraction for the local X-ray groups and clusters Giodini et al. 2009) and assume that the stellar populations evolve according to the "fossil" model of Nagamine et al. (2006).
Following Ostriker et al. (2005), a fraction of the rest mass energy of the stars, $\epsilon_{f} M_{*} c^{2}$, is put back into the gas due to energy feedback from SNe and AGN, where $\epsilon_{f}$ is a free parameter. The model also accounts for the transfer of energy from dark matter to gas by dynamical heating by infalling substructures, determined by the product of the total binding energy of the halo $\left|E_{D M}\right|$ and a free parameter $\epsilon_{D M}$. Finally, we take into account the non-thermal pressure support due to random gas motions as seen in hydrodynamical simulations (e.g., Kav et al. 2004; Rasia et al. 2004; Lau et al. 2009; Battaglia et al. 2010; Nelson et al. 2011). We assume the non-thermal pressure to be a certain fraction of the total 

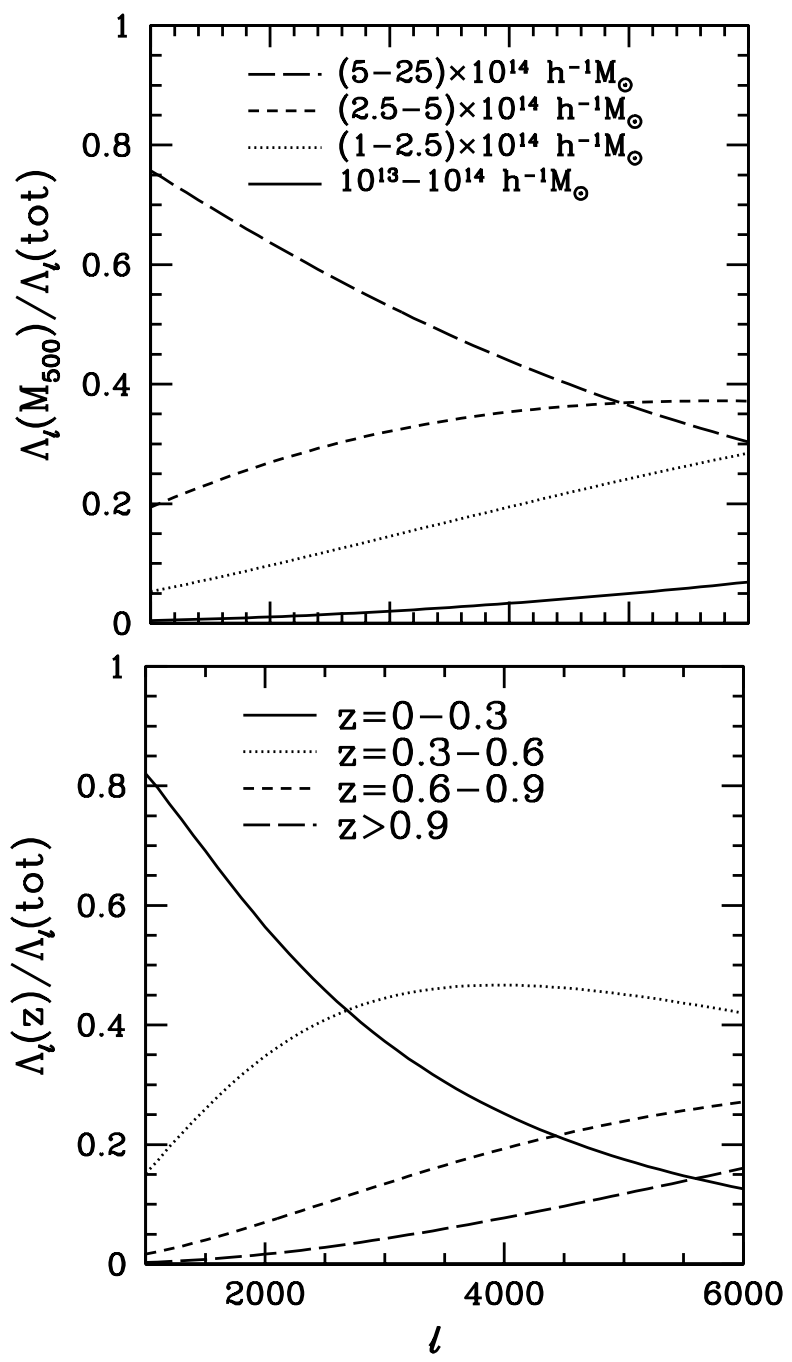

FIG. 2.- Contribution to the SZ skewness spectrum of objects within a given mass range (top) and redshift range (bottom) range. We show the ratio of the skewness spectrum in a mass/redshift range to the total skewness spectrum.

pressure and a power law in radius,

$$
\frac{P_{\mathrm{nt}}}{P_{\mathrm{tot}}}(z)=\alpha(z)\left(\frac{r}{R_{500}}\right)^{n_{\mathrm{nt}}},
$$

where $\alpha(z)=\alpha_{0} f(z), \alpha_{0}$ is the ratio of non-thermal to total pressure at $R_{500}$, the radius at which the spherical overdensity of the cluster is 500 times the critical density of the universe (enclosing mass $\left.M_{500}\right) . f(z)=$ $\min \left[(1+z)^{\beta},\left(4^{-n_{\mathrm{nt}}} / \alpha_{0}-1\right) \tanh (\beta z)+1\right], n_{\mathrm{nt}}$ and $\beta$ are the radial and redshift dependence of the non-thermal pressure support, respectively. We set $n_{\mathrm{nt}}=0.8$, motivated by comparisons to hydrodynamical simulations (S10, Battaglia et al. 2011), leaving $\beta$ as a free parameter.

In summary, our ICM model has five free parameters $A_{C}, \epsilon_{f}, \epsilon_{\mathrm{DM}}, \alpha_{0}$, and $\beta$. Since the key observable for the $\mathrm{SZ}$ effect is the pressure profile, we use measurements of the pressure profiles by Arnaud et al. (2010) based on 31 massive $\left(M_{500}>10^{14} h^{-1} M_{\odot}\right)$, low redshift $(z \lesssim 0.2)$ clusters. Arnaud et al. (2010) showed that the pressure
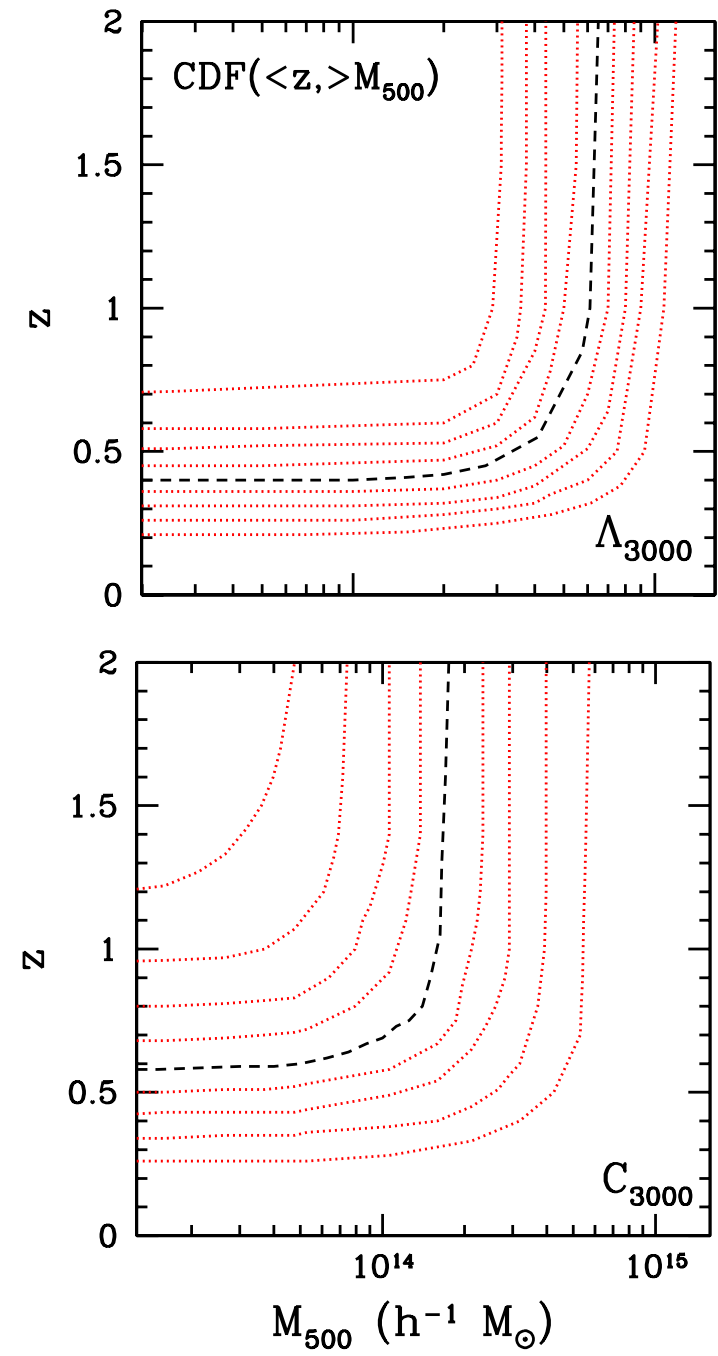

FIG. 3.- Top panel: the distribution of the skewness spectrum over mass and redshift at $\ell=3000$. At $M_{500}=5 \times 10^{13} h^{-1} M_{\odot}$, the lines show the cumulative distribution function, $\operatorname{CDF}(>\mathrm{M}$, $<\mathrm{z})=0.1$ to 0.9 from bottom to top with a step of 0.1 . Bottom panel: the distribution of the SZ power spectrum at $\ell=3000$ (for comparison with the skewness distribution). The lines represent similar quantities as that in the top panel.

profiles in their sample adheres to a universal form with $\sim 20 \%$ scatter. Figure 1 shows the pressure profile fit to the Arnaud et al. (2010) data along with our predictions for a typical cluster of mass $M_{500}^{\mathrm{HSE}}=3 \times 10^{14} h^{-1} M_{\odot}$ at $\mathrm{z}=0$, where $M_{500}^{\mathrm{HSE}}$ is a cluster mass estimated assuming the hydrostatic equilibrium (see S10 for more discussions). Each of the 5 parameters are varied over a wide range, including some extreme cases with a very high feedback parameter or zero non-thermal pressure.

The parameter $\epsilon_{f}$ determines the amount of nongravitational ('feedback') energy injected into the ICM. High levels of feedback inflates the gas distribution, lowering the pressure in the inner region while boosting it in the cluster outskirts. For cluster-mass halos, the feedback energy is a small fraction of the binding energy and has a minimal effect on the pressure profile. The upper-left panel of Figure 1 shows how $\epsilon_{f}$ changes the pressure profile in a cluster-mass halo at $z=0$. 
Increasing $\epsilon_{f}$ by factor of $\sim 100$ changes the pressure profile by few percent at radii $R<R_{500}$ and increases to about $30 \%$ at $3 R_{500}$. This is in qualitative agreement with the findings from hydrodynamical simulations including AGN feedback which indicate the impact of AGN feedback is more pronounced in groups than in clusters (Bhattacharva et al. 2008; Puchwein et al. 2008: Sijacki et al. 2007; Teyssier et al. 2011; McCarthy et al. 2010; Battaglia et al. 2010, 2011; Short et al. 2012). We note however that our feedback model is highly simplistic and does not capture the full detail of AGN feedback as implemented in hydrodynamical simulations (for example, energy injection via jets).

$\epsilon_{D M}$ determines the fraction of the total dark matter energy transferred to the ICM during mergers. Clusters frequently undergo mergers and hence $\epsilon_{D M}$ may have a substantial effect on the ICM physics of clusters. As shown in Figure 1 (top-left), $\epsilon_{D M}$ changes the pressure profile by $20-30 \%$ over the entire radius range as $\epsilon_{D M}$ varies from $0-0.2$.

The normalization of the concentration-mass relation $\left(A_{c}\right)$ determines the characteristic radius of an NFW dark matter profile, approximately corresponding to the radius at which the slope changes from -1 to -3 . The upper-right panel of Figure 1 shows the change in the thermal pressure profile as $A_{c}$ varies from $0.8-1.2$. Increasing the concentration deepens the central potential which draws the gas towards the cluster core, steepening the pressure profile. The impact of increasing halo concentration provides a similar (although opposite) effect to that of energy feedback.

The nonthermal pressure parameter, $\alpha_{0}$, determines the fraction of the total gas pressure contributed by random gas motions. As $\alpha_{0}$ is varied is the thermal pressure fraction adjusts accordingly such that the total gas pressure is fixed. As shown in Figure 1 (bottom-left), the thermal pressure profile changes by about $60 \%$ at $R_{500}$ as $\alpha_{0}$ varies from $0-0.3$. $\beta$ determines the redshift evolution of the non-thermal pressure fraction. S10 obtained an estimate for $\beta$ by comparing the redshift 0 and 1 outputs of the simulations of Lau et al. (2009), finding that the best fit value for $\alpha_{0}$ increases from $0.18-0.26$ implying $\beta=0.5$. The bottom-right panel in Figure 1 shows the pressure profile of a cluster at $\mathrm{z}=0.5$ and how $\beta$ changes the thermal pressure profile. A positive value of $\beta$ increases the amount of non-thermal pressure at higher redshift and hence decreases the thermal pressure as redshift increases. The pressure profile decreases by about $20 \%$ as $\beta$ varies from -1 to 1 .

Overall, we find that the range $\epsilon_{f}<10^{-6}, \epsilon_{D M}=$ $0.04-0.06, A_{c}=0.8-1.1$, and $\alpha_{0}=0.14-0.24$ to be in agreement with the Arnaud pressure profile within the uncertainty (see Figure 11). Note that, as described in S10, the constraints on $\epsilon_{f}<10^{-6}$ and $\epsilon_{D M}=0.04-0.06$ mainly come from the gas fractionmass relation of Vikhlinin et al. (2006). We also find the entropy scaling relation (e.g., entropy - X-ray temperature relation) predicted by our ICM model to be in agreement with the data of Pratt et al. (2010) within the ranges of the ICM parameters. This illustrates that the current observations can indeed constrain the ICM parameter space significantly.

\section{THE SZ SKEWNESS SPECTRUM}

\begin{tabular}{|c|c|c|c|}
\hline \hline $\begin{array}{c}\text { Mass Range } \\
M_{500}\left[h^{-1} M_{\odot}\right]\end{array}$ & $\begin{array}{c}\text { power } \\
\%\end{array}$ & z-range & $\begin{array}{c}\text { power } \\
\%\end{array}$ \\
\hline \hline$<10^{14}$ & 3 & $0-0.3$ & 38 \\
$(1-2.5) \times 10^{14}$ & 15 & $0.3-0.6$ & 45 \\
$(2.5-5) \times 10^{14}$ & 30 & $0.6-0.9$ & 12 \\
$(5-25) \times 10^{14}$ & 52 & $>0.9$ & 5 \\
\hline \hline
\end{tabular}

TABLE 2

Distribution OF THE SKEWNESS SPECTRUM OVER DIFFERENT MASS RANGE AT $\ell \sim 3000$.

\subsection{Mass and redshift contributions}

Figure 2 shows the fraction of skewness spectrum signal contributed by different ranges of halo mass and redshift. At $\ell \sim 3000$, galaxy groups $\left(M_{500}<10^{14} h^{-1} M_{\odot}\right)$ source only $5 \%$ of the signal, while lower mass clusters contribute about $20 \%$. The majority of the signal comes from the massive clusters. The distribution of the skewness spectrum is tabulated in Table 2 over different mass and redshift ranges. We also calculate the signal expected from clusters that are below the detection threshold of the current generation of SZ surveys. Following Benson et al. (2011); Vanderlinde et al. (2010), we choose the SPT mass threshold of $M_{500} \sim$ $2.5 \times 10^{14} h^{-1} M_{\odot}$, roughly corresponding to the detection significance of $5 \sigma$. The skewness spectrum gets only $20 \%$ (at $\ell=1500$ ) to $40 \%$ (at $\ell=6000$ ) of the signal from clusters with $M_{500} \lesssim 2.5 \times 10^{14} h^{-1} M_{\odot}$. In contrast, the SZ power spectrum gets about two-thirds of the signal from groups and clusters with $M_{500} \lesssim 2.5 \times 10^{14} h^{-1} M_{\odot}$.

Figure [3, top panel shows the cumulative fractional distribution of the skewness spectrum for halos with mass $<M_{500}$ and redshift $>z$, from $10-90 \%$ of the signal (topleft to bottom-right) at $\ell=3000$. In terms of the redshift distribution, about $90 \%$ of the skewness spectrum signal comes from $\mathrm{z}<1$. The bottom panel of Figure 3 shows the distribution of the SZ power spectrum, showing that a significant fraction of the thermal SZ power comes from high-z, low-mass objects (i.e., about $40 \%$ of the power coming from $z>0.7$ and $\left.M_{500}<10^{14} h^{-1} M_{\odot}\right)$ where the ICM properties are still very uncertain. In comparison, the SZ skewness spectrum signal is dominated by low-z, massive clusters.

\subsection{Astrophysical Uncertainties}

We now evaluate how robust the predicted skewness spectrum is to uncertainties in the underlying ICM model parameters. Figure 4 shows the variation of the SZ skewness spectrum over the range of ICM parameters given in Table 1 and plotted in Figure 1] As discussed in Section 3. $\epsilon_{f}$ does not have a significant effect on the massive clusters that dominate the skewness spectrum. Consequently, as shown in the top-left panel of Figure 1] the skewness spectrum varies only by $14 \%$ as $\epsilon_{f}$ varies from $(1-100) \times 10^{-7}$. On the other hand, the pressure profiles of massive clusters vary substantially with $\epsilon_{D M}$, and the skewness spectrum varies by about $40 \%$ as $\epsilon_{D M}$ is varied from 0 to 0.2 . In contrast, the power spectrum receives a significant contribution from a wider range in mass, $M_{500}=10^{13}-10^{15} h^{-1} M_{\odot}$, especially at high $\ell$. As a result, $\epsilon_{f}$ shows more variations at $\ell=8000$ (group scales) than at $\ell=2000$ (cluster scales, see Fig. 7 of S10). 


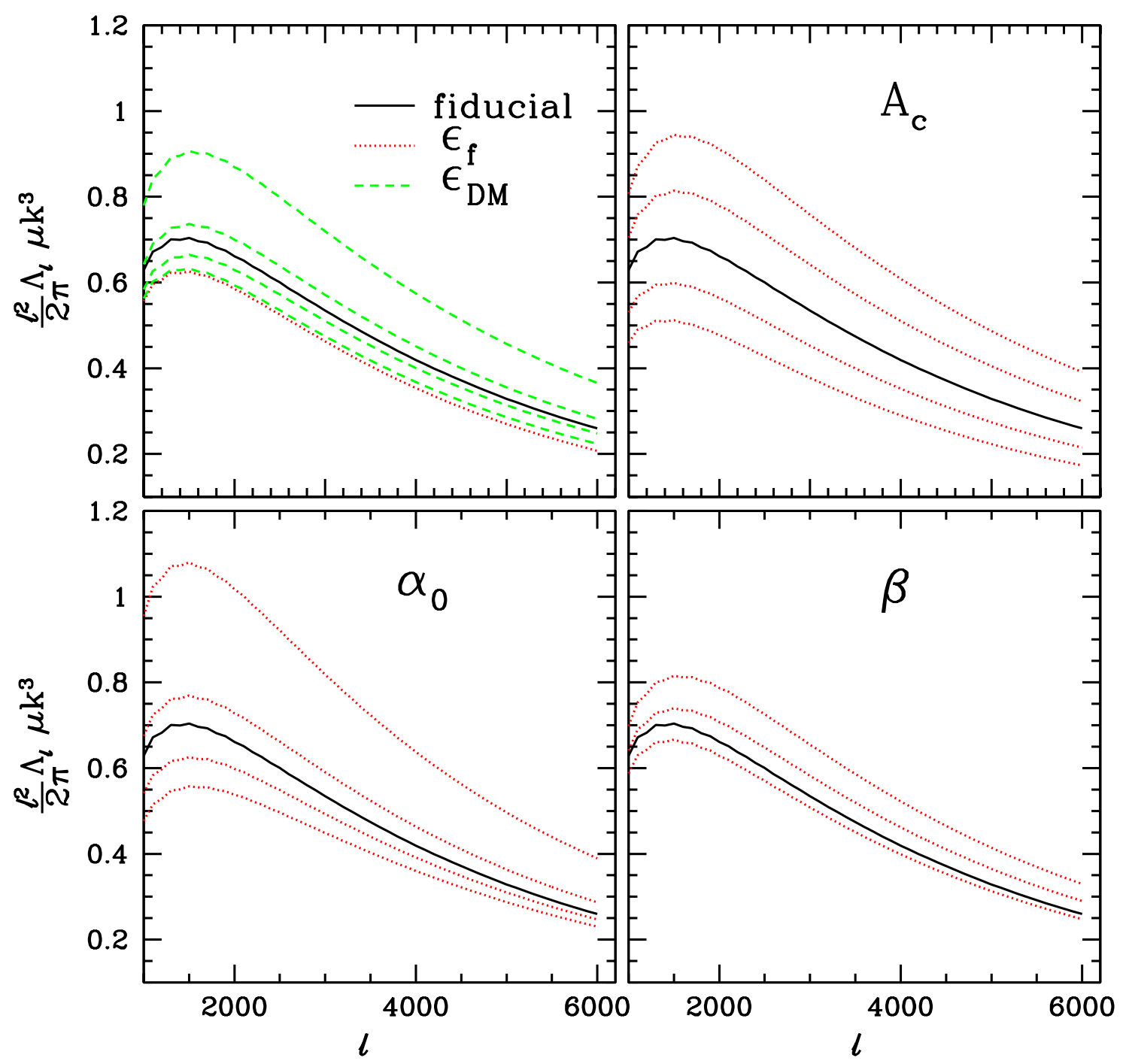

FIG. 4.- Dependence of the SZ skewness spectrum on the cluster gas physics. The solid line indicates the fiducial model with $\epsilon_{f}=$ $4 \times 10^{-7}, \epsilon_{D M}=0.05, A_{c}=1.0, \alpha_{0}=0.2$, and $\beta=0.5$. The dotted and dashed lines represent the skewness spectrum predicted by our ICM model for a range of ICM parameter values. The top-left panel shows the impact of $\epsilon_{f}$ (red dotted line: $10^{-5}$ and black solid line: $4 \times 10^{-7}$ ) and $\epsilon_{D M}$ (green dashed lines: $0.0,0.04,0.06$, and 0.2 from top to bottom); the top-right panel shows the change with $A_{c}$ $\left(0.8,0.9,1.0,1.1,1.2\right.$ from bottom to top); the bottom-left panel shows the variations with $\alpha_{0}(0.0,0.14,0.2,0.24,0.3$ from top to bottom); and the bottom-right panel shows the variation with $\beta$ ( $-1,0,0.5,1$ from top to bottom).

Increasing the normalization of the concentration-mass relation $\left(A_{c}\right)$ deepens the potential well of the clusters which steepens the pressure profiles. The top-right panel of Figure 4 shows the change in the skewness spectrum is about $\pm 30 \%$ for a $\pm 20 \%$ change in $A_{c}$ (compared to the power spectrum where the variation is $\sim 20 \%$ ). This can be understood from the fact that the skewness amplitude is $\propto$ (power spectrum $)^{1.5}$, so a $20 \%$ change in power spectrum corresponds to a $30 \%$ change in the skewness spectrum. Also note that in contrast to the case of $\epsilon_{f}$, the effect of $A_{c}$ is not significantly mass-dependent.

The lower-left panel shows the effect on the skewness spectrum of varying the non-thermal pressure parameter $\alpha_{0}$. The skewness spectrum changes by about $70 \%$ across the range $\alpha_{0}=0-0.3$. As the SZ skewness spectrum gets most of its signal from massive clusters at $z \lesssim 0.4$, the uncertainty in the z-evolution of the non-thermal pressure $(\beta)$ contributes only about $20 \%$ uncertainty to the SZ skewness spectrum. The SZ power spectrum, on the other hand gets its signal from high-z objects and as a result $\beta$ adds $\sim 30-40 \%$ uncertainty in the SZ power spectrum (bottom-right panel of Figure 7 in S10).

Figure 5 shows the astrophysical uncertainties in the shaded area around the fiducial model. Here, the total uncertainty is computed by adding the uncertainties (calibrated by the observed pressure profile and discussed in detail in Section (3) in each ICM parameter in quadrature. On average, about $75-80 \%$ of the skewness spectrum signal arises from massive lower redshift objects; i.e., $M_{500} \gtrsim 5 \times 10^{14} h^{-1} M_{\odot}$ at $\langle z\rangle 0.4$, and hence is less sensitive to the variation in ICM parameters than the power spectrum (c.f. Figure 7 in S10). Figure 5 shows 


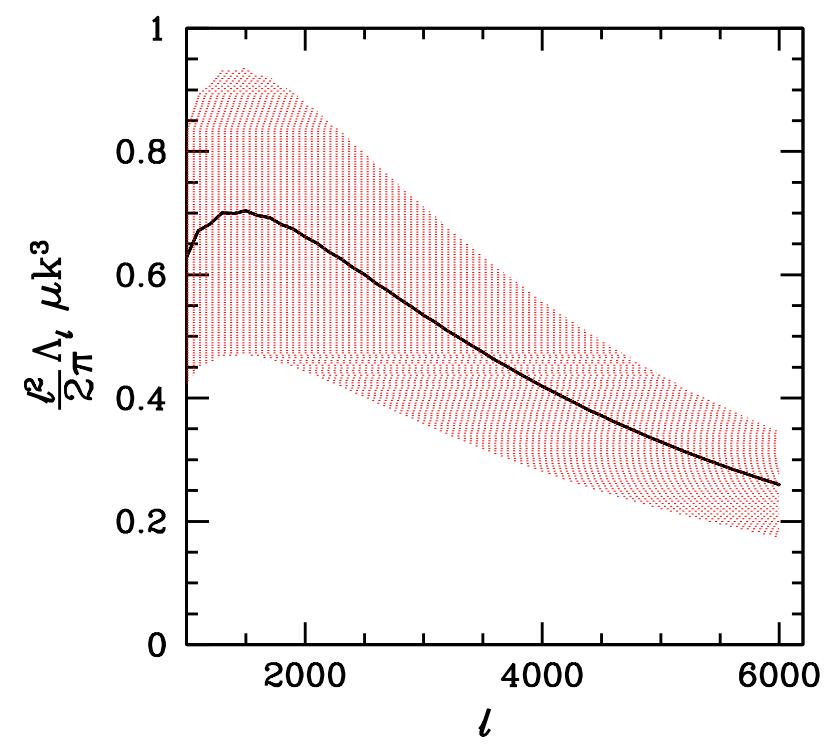

FIG. 5.- Astrophysical uncertainties in the SZ skewness spectrum. The solid line represents the fiducial model and the shaded region indicates astrophysical uncertainty for the ICM parameter range calibrated by the observed pressure profile (see Section 3). At $l=3000$, the astrophysical uncertainties are $\sim 33 \%$.

the gas physics uncertainty in the skewness spectrum to be about $33 \%$. We also provide the theoretical prediction of the skewness spectrum for the fiducial values of cosmological and ICM parameters.

At this point, it is worth commenting on effects of several simplifying assumptions in our model. First, we assumed a constant $\Gamma=1.2$. However, recent work suggests that $\Gamma$ could be radially dependent, especially at large radii $\left(r<R_{\mathrm{vir}}\right)$. However, the bulk $(\approx 95 \%)$ of the skewness signal arises from $r<R_{\text {vir }}$, and $\Gamma \approx 1.14-1.3$ over the redshift range $z=0-1$ (Battaglia et al. 2011). We therefore find that the skewness spectrum changes by about $\pm 10-15 \%$ in the specified range of $\Gamma$. Also, there is uncertainty in the number of baryons converted into stars in clusters, since this is only constrained by redshift zero observations. Secondly, the redshift dependence of the model assumes that evolution of the stellar fraction is the same for all galaxies, although it is possible that the star fraction evolve differently in a cluster environment. In order to assess the impact of star formation model on the skewness spectrum, we considered a no evolution model and found that the difference between the "no-evolution" model and the fiducial model (namely the "fossil" model of Nagamine et al. (2006)) is negligible. Finally, recent work on the thermal SZ power spectrum have shown that variations about a mean profile may produce up to a $15 \%$ excess in the spectrum at $\ell \sim 3000$ (e.g., Battaglia et al. 2011). This variation is non-Gaussian and radially-dependent and can lead to the enhancement of the bispectrum amplitude.

\subsection{The relation between the power spectrum and the skewness spectrum amplitude}

Given a measurement of the SZ skewness spectrum amplitude, we can predict the expected amplitude of the thermal SZ power spectrum, namely the $B_{\mathrm{tSZ}}-A_{\mathrm{tSZ}}$

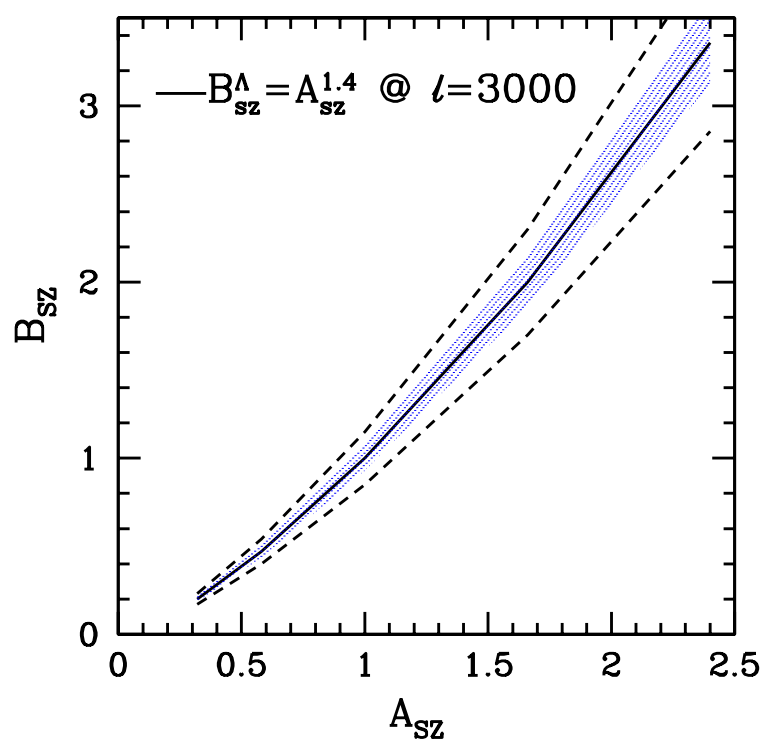

FIG. 6.- Scaling of $B_{\mathrm{tSZ}}-A_{\mathrm{tSZ}}$ for the skewness spectrum when $\sigma_{8}$ varies from 0.7 to 0.9 . The relations are relatively robust to the changes in gas physics and vary by only $\sim 7 \%$ (shaded area) for the allowed ICM parameter range calibrated by the observed pressure profile (see Section 3). The dashed lines indicate the uncertainty range bracketed by the two extreme gas physics scenarios.

\begin{tabular}{|c|c|}
\hline \hline$\ell$ & $\ell^{2} /(2 \pi) \Lambda(l) \mu \mathrm{K}^{3}$ \\
\hline \hline 1000 & 0.66 \\
2000 & 0.69 \\
3000 & 0.56 \\
4000 & 0.44 \\
5000 & 0.35 \\
6000 & 0.28 \\
\hline \hline
\end{tabular}

TABLE 3

THE THEORETICAL PREDICTION OF THE SKEWNESS SPECTRUM FOR THE FIDUCIAL VALUES OF ICM AND COSMOLOGY PARAMETERS.

relation. Recall that the power spectrum and the skewness spectrum are proportional to the square and the cube of the pressure profile, respectively. If we assume the skewness spectrum and the power spectrum have the same mass and redshift distribution, then any changes in gas physics affects the skewness spectrum and (power spectrum $)^{1.5}$ in the same way. However, as shown in Sec 4.1, the skewness spectrum depends mostly on the massive clusters at intermediate redshift, while the power spectrum receives a substantial contribution from higher redshift galaxy groups. Furthermore, the cluster physics affect the gas properties in lower mass structures more than in clusters. Therefore, the $B_{\mathrm{tSZ}}-A_{\mathrm{tSZ}}$ relation is expected to deviate from the above relation and depends on both cosmology and cluster astrophysics.

To investigate the dependence of the $B_{\mathrm{tSZ}}-A_{\mathrm{tSZ}}$ relation on cluster physics and cosmology, we vary $\sigma_{8}$ from $0.7-0.9$ in steps of 0.05 and compute the $B_{\mathrm{tSZ}}-A_{\mathrm{tSZ}}$ relation for each value of $\sigma_{8}$ for our fiducial gas physics model. Figure 6 shows that the skewness spectrum amplitude is proportional to the power spectrum amplitude,

$$
B_{\mathrm{tSZ}}(\ell=3000)=A_{\mathrm{tSZ}}^{1.4}(\ell=3000) .
$$

This is shallower than the expected $A_{\mathrm{tSZ}}^{1.5}$ behavior, primarily because the skewness spectrum signal comes from 

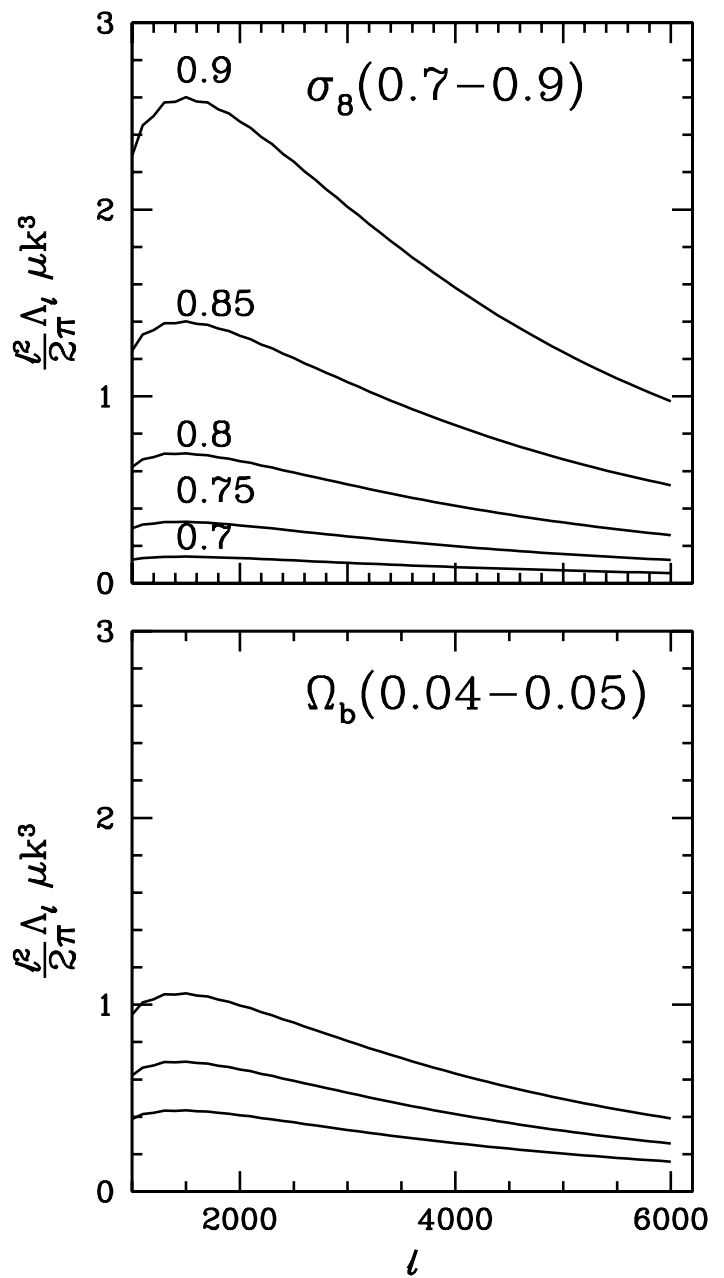

Fig. 7.- Dependence of the skewness spectrum on $\sigma_{8}$ and $\Omega_{b}$. The fiducial values are $\Omega_{b}=0.045$ and $\sigma_{8}=0.8$. The range of parameters are indicated in the top-right corner of each panel. The parameter values increase from bottom to top.

more massive clusters which are not point-like at the angular scales equivalent to $\ell \sim 3000$. We thus study the radial dependence of the skewness spectrum signal and find that less than $5 \%$ signal comes from $[1-2] R_{\text {vir }}, 20 \%$ comes from $[0.5-1] R_{\text {vir }}, 60 \%$ from $[0.2-0.5] R_{\text {vir }}$, the rest $15 \%$ from $\leq 0.2 R_{\text {vir }}$. Thus a simple scaling of the pressure normalization with mass cannot explain the $B_{\mathrm{tSZ}}-A_{\mathrm{tSZ}}$ relation. Next we investigate the variation of the scaling relation with the ICM parameters. Within the range of the ICM parameters allowed by the observed pressure profile, we find the $B_{\mathrm{tSZ}}-A_{\mathrm{tSZ}}$ relation is robust and the overall uncertainty does not vary by more than $7 \%$ (indicated by shaded area in Figure 6). The tight scaling arises because both the power spectrum and the skewness spectrum depend on the pressure profile. As a result, the amplitudes of the two spectra varies in similar ways as we vary our model parameters.

To check the robustness of the relation further, we consider two extreme gas physics scenarios, such that the $\mathrm{SZ}$ power spectrum varies from $2.5-10 \mu \mathrm{K}^{2}$ (roughly by factor of $\sim 4$ ) which is roughly twice the theoretical uncertainty assumed in the current SZ power spectrum analysis (Reichardt et al. 2011). The first case is where the pressure profile amplitude is maximally suppressed by high feedback and non-thermal pressure and low concentration: $\epsilon_{f}=10^{-5}, \epsilon_{D M}=0.1, \alpha_{0}=0.3$, and $A_{c}=0.8$. The second case other case consists of the parameter choice that predicts a higher amplitude: $\epsilon_{f}=\epsilon_{D M}=\alpha_{0}=0.0$ and $A_{c}=1.2$. We find that even for the two extreme gas physics scenarios the $A_{\mathrm{SZ}}-B_{\mathrm{tSZ}}$ has an overall uncertainty of only by $\sim 15 \%$ (indicated by dashed lines in Fig (6). The slope of the relation changes from 1.33 to 1.47 between these two cases. This is encouraging as using the skewness spectrum data we can measure $B_{\mathrm{tSZ}}$, then use this theoretical relation to derive $A_{\mathrm{tSZ}}$.

The relation in Eq. 17 implies the skewness spectrum amplitude varies as $\sigma_{8}^{11-12}$. We also investigate how the skewness spectrum varies with other cosmological parameters. Figure 7 shows the variation of the skewness spectrum with $\sigma_{8}$ and $\Omega_{b}$. Each panel shows how each cosmological parameter changes the skewness spectrum while other parameters are fixed at their respective fiducial values. The figure shows that the skewness spectrum depends very sensitively on $\sigma_{8}$, and the amplitude varies as $B_{\mathrm{SZ}}^{\Lambda} \propto \sigma_{8}^{11.6}$, as expected. The variations with $\Omega_{b}$ is moderate, while $\Omega_{m}, h, w_{0}$, and $n_{s}$ show much smaller variation. We find the SZ amplitude for the skewness spectrum follows the scaling (at $\ell=3000)$,

$$
\begin{aligned}
B_{\mathrm{tSZ}} & \propto\left(\frac{\sigma_{8}}{0.8}\right)^{11.6}\left(\frac{\Omega_{b}}{0.045}\right)^{4.1}\left(\frac{h}{0.71}\right)^{2.5}\left(\frac{w_{0}}{-1.0}\right) \\
& \times\left(\frac{n_{s}}{0.97}\right)^{-1.5}\left(\frac{\Omega_{m}}{0.27}\right)^{-0.46} \cdot
\end{aligned}
$$

Note that not all cosmological parameters follow a simple $B_{\mathrm{tSZ}}=A_{\mathrm{tSZ}}^{1.4}$ relation. The difference in scaling is due to the different range of masses in the mass function contributing to the skewness spectrum and the power spectrum. Note that we ignore the cosmology dependence of the concentration-mass relation (Bhattacharya et al. 2011a) in our study which can lead to a slightly different scaling of the skewness spectrum amplitude with cosmology. We provide the theoretical prediction of the skewness spectrum in Table 3 for our fiducial cosmology and gas parameters.

\section{DETECTABILITY}

We now assess the detectability of the SZ skewness spectrum in the presence of other secondary CMB anisotropy signals and extra-galactic foregrounds. Other than the thermal SZ effect, point sources (dusty, starforming galaxies (DSFGs), and radio sources) will also contribute to the skewness spectrum signal. The main difference is while the thermal SZ effect contribute a negative bispectrum, the point sources contribute a positive signal (Rubiño-Martín \& Sunyaev 2003). Assuming that the clustered component of the DSFGs follows the Gaussian distribution, the skewness spectrum of the correlated DSFG is zero. As mentioned before, the kinetic SZ follow approximately Gaussian distribution and is thus assumed to have negligible skewness. We also ignore a possible skewness spectrum signal arising from the correlation between the SZ effect and the DSFG sources.

\subsection{Noise Estimates}



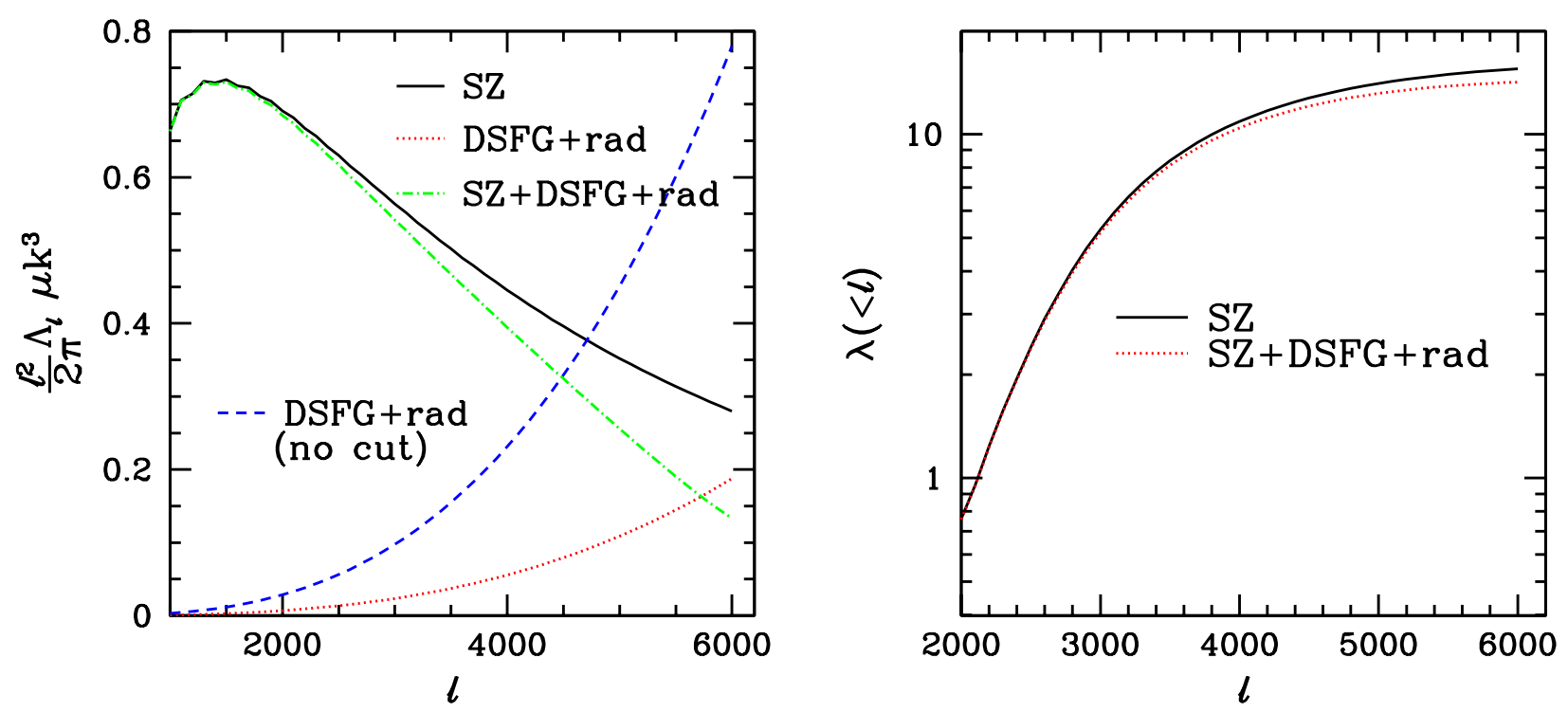

FIG. 8.- Detectability of the SZ skewness spectrum at $150 \mathrm{GHz}$. The left panel shows the SZ (black solid) and the point sourcesDSFG+radio skewness spectrum (red dotted line). Also shown are the total skewness spectrum: SZ+DSFG+radio (green thin dot-dashed) and the DSFG+radio skewness spectrum if no point sources are removed from the map (blue short dashed line). The right panel shows the signal-to-noise integrated to a certain $\ell$ of the SZ skewness spectrum (black solid), the total- SZ+DSFG+radio (red dotted). We assume an SPT-like survey with sky coverage of $2500 \mathrm{deg}^{2}, 18 \mu \mathrm{K}$-arcmin, $1.2 \operatorname{arcmin}$ at $150 \mathrm{GHz}$.

The noise of the total skewness spectrum, assuming the primary contribution to the variance of the bispectrum comes from the power spectrum of the CMB sky, is given by Komatsu \& Spergel (2001)

$$
N^{2}\left(\ell_{1} \ell_{2} \ell_{3}\right)=C\left(\ell_{1}\right) C\left(\ell_{2}\right) C\left(\ell_{3}\right) \Delta_{\ell_{1} \ell_{2} \ell_{3}},
$$

where $C(\ell)$ is the total power spectrum that includes contributions from (1) the SZ power spectrum computed using Eq.2. (2) the lensed CMB power spectrum computed using CAMB for the fiducial cosmology (Lewis et al. 2000), (3) the noise due to the finite resolution of an experiment, and (4) point sources, including dusty starforming galaxies (DSFG) and radio sources. Note that $\Delta_{\ell_{1} \ell_{2} \ell_{3}}=6$ if all three $\ell_{\mathrm{s}}$ are equal, $\Delta_{\ell_{1} \ell_{2} \ell_{3}}=2$ if two of the three $\ell \mathrm{s}$ are equal and $\Delta_{\ell_{1} \ell_{2} \ell_{3}}=1$ if all three $\ell \mathrm{s}$ are different. We describe the estimate of (3) and (4) in more detail below.

The noise due to the finite resolution of the experiment is given by Knox (1995); Jungman et al. (1996)

$$
N_{b}(\ell)=w^{-1} \exp \left[\frac{\ell^{2} \theta^{2}}{8 \log 2}\right],
$$

where $w^{-1}=\left[\sigma_{\text {pix }} \theta / T_{\mathrm{CMB}}\right]^{2}$ is the weight per unit solid angle, $\sigma_{\text {pix }}$ is the noise per pixel, $\theta$ is the FWHM of the instrument in radians, $T_{\mathrm{CMB}}$ is the $\mathrm{CMB}$ temperature. Eq. 19 gives the expression for noise in the full sky limit. For the fractional coverage, Eq. 19 is multiplied by $f_{\text {sky }}^{-3 / 2}$, where $f_{\text {sky }}$ is the fraction of the sky covered by the assumed surveys. Here we consider an SPT like survey with $f_{\text {sky }}$ given by $2500 \mathrm{deg}^{2}$ sky coverage, $\sigma_{\text {pix }}$ is $18 \mu \mathrm{K}$-arcmin at $150 \mathrm{GHz}$ with the pixel size set by a Gaussian beam of FWHM=1.2 arcmin. The powers of the unresolved DSFG sources at $150 \mathrm{GHz}$ are given by $5.25 \times 10^{-6} \mu \mathrm{K}^{2}$ (Poisson) and $4.4 \times 10^{-6}(\ell / 3000)^{-1.2} \mu \mathrm{K}^{2}$ (clustered), and that of the radio sources is $9 \times 10^{-7} \mu \mathrm{K}^{2}$
(Poisson) at $150 \mathrm{GHz}$ (Reichardt et al. 2011).

\subsection{Point Source Bispectrum}

The DSFG population comprises of a clustered component and a Poisson component, both of which contribute to the point source power spectrum. We assume that the clustered component produces Gaussian fluctuations and hence does not contribute to the bispectrum of the point sources. We thus consider the bispectrum contribution only from the Poisson component of the DSFGs and the radio galaxies. The Poisson component of the DSFG typically comprises of two populations: (1) the bright point sources which are detected by the CMB experiments (Vieira et al. 2010; Marriage et al. 2011) and (2) the faint sources which follow a shallower distribution compared to the bright sources, and are undetected by the current $\mathrm{CMB}$ experiments but contribute a significant portion of the arcminute scale DSFG power measured in the $\mathrm{CMB}$ experiments. Both populations are expected to contribute to the total point source bispectrum.

Given the DSFG flux number counts $d N / d S$, we can write the bispectrum of DSFGs as

$$
\frac{b_{\mathrm{DSFG}}}{g^{3}\left(x_{\nu}\right)}=\int_{0}^{S_{\mathrm{min}}} d S S^{3} \frac{d N}{d S}=\frac{N_{\mathrm{DSFG}}\left(>S_{\mathrm{min}}\right)}{3 / \beta_{\mathrm{DSFG}}-1} S_{\mathrm{min}}^{3}
$$

where the flux number counts is assumed to vary as $d N / d S \propto S^{-\beta-1}, \quad S_{\min }$ is the flux cut above which all sources are detected and removed from the CMB maps, $N_{\mathrm{DSFG}}\left(>S_{\mathrm{min}}\right)$ is the number density (per unit solid angle) of the DSFG with $S>S_{\min }$, $g\left(x_{\nu}\right)=1 /\left(65.55 \mathrm{MJy} \mathrm{sr}^{-1}\right)\left[\sinh \left(x_{\nu} / 2\right) / x_{\nu}^{2}\right]^{2}$, and $x_{\nu}=$ $\nu /(56.8 \mathrm{GHz})$. Similarly, we define the bispectrum of the radio sources in terms of the radio source power spectrum 
as

$$
\frac{b_{\mathrm{rad}}}{g^{3}\left(x_{\nu}\right)}=\int_{0}^{S_{\mathrm{min}}} d S S^{3} \frac{d N}{d S}=\frac{N_{\mathrm{rad}}\left(>S_{\mathrm{min}}\right)}{3 / \beta_{\mathrm{rad}}-1} S_{\mathrm{min}}^{3} .
$$

We assume all point sources above $5 \sigma$ at $150 \mathrm{GHz}$ are detected and removed (masked). For the SPT $150 \mathrm{GHz}$ band, this corresponds to $S_{\min }=6.4 \mathrm{mJy}$ (Vieira et al. 2010). We adopt the number density of the DSFG to be $N_{\mathrm{DSFG}}=0.3 \mathrm{deg}^{-2}$ (Negrello et al. 2007) and $\beta_{\mathrm{DSFG}}=$ 1.93 for the total (bright + faint) DSFG population to be consistent with the total DSFG power spectrum measured in the CMB surveys (e.g., Reichardt et al. 2011). For the radio population we adopt $N_{\text {rad }}\left(>S_{\min }\right)=$ $1.29 \mathrm{deg}^{-2}$ and $\beta_{\mathrm{rad}}=1.03$, which is consistent with the $150 \mathrm{GHz}$ source counts in Vieira et al. (2010). The skewness spectra of the radio sources and DSFGs are calculated the same way as the SZ skewness spectrum by plugging Eqs. 21 and 22 in Eq. 10.

The skewness spectrum for the various components at $150 \mathrm{GHz}$ are shown in the left panel of Figure 8. Provided the point sources that are detected above $5 \sigma$ in the SPT band-power are removed, the DSFG and radio population make negligible contribution to the skewness spectrum at $\ell \lesssim 4000$. Beyond this scale the skewness spectra of point sources and SZ effect become comparable. If no point sources are removed, then the skewness spectrum of the point sources (DSFG+radio) is a factor of 4-5 higher compared to the case with point source removal and becomes the dominant signal at $\ell>5000$. Since the point sources and the SZ bispectrum have opposite signs, the total skewness spectrum ( $\mathrm{SZ}+$ point sources) is smaller than the SZ skewness spectrum alone, especially at higher- $\ell$ where the point source bispectrum becomes non-negligible.

\subsection{Signal-to-Noise}

Finally, using Eq. 7, we define the signal-to-noise (S/N) in terms of the full-sky bispectrum. The $\mathrm{S} / \mathrm{N}$ integrated to a certain $\ell$ (Komatsu \& Spergel 2001; Hu 2000), $\lambda(<$ $\ell)$ or $\lambda_{\ell}$ can be written as

$$
\lambda(<\ell)=\sqrt{\sum_{\ell_{1} \ell_{2} \ell_{3}} \frac{B^{2}\left(\ell_{1} \ell_{2} \ell_{3}\right)}{N^{2}\left(\ell_{1} \ell_{2} \ell_{3}\right)}},
$$

where $l_{3} \leq l_{2} \leq l_{1} \leq l$. The right panel of Figure 8 shows the total $\mathrm{S} / \mathrm{N}$ for the $\mathrm{SZ}$ skewness spectrum. For a $2500 \mathrm{deg}^{2} \mathrm{SPT}$ like survey at $150 \mathrm{GHz}$, the integrated $\mathrm{S} / \mathrm{N}$ for the $\mathrm{SZ}$ skewness spectrum is $\approx 16$. Note that the total ( $\mathrm{SZ}+$ point source) skewness spectrum is lower than the SZ skewness spectrum alone, because these two spectra have opposite sign. We find the $\mathrm{S} / \mathrm{N}$ of the total skewness spectrum is only $10 \%$ smaller than the SZ only case.

\section{SUMMARY AND DISCUSSIONS}

We investigated the SZ bispectrum and the prospects of its detection with the current and upcoming CMB surveys. We define a skewness spectrum of the SZ effect which is a sum over all possible triangle configurations over the two smaller sides and expressed as a function of the largest side. About half of the SZ skewness spectrum signal arises from massive $\left(M_{500} \gtrsim 6.5 \times 10^{14} h^{-1} M_{\odot}\right)$ clusters in the local universe $(z \lesssim 0.4)$. This is in contrast to the SZ power spectrum whose signals come primarily from high-z, low-mass galaxy groups $\left(M_{500}<\right.$ $2 \times 10^{14} h^{-1} M_{\odot}$ and $\left.z \gtrsim 0.6\right)$ that have little observational constraints at present. The skewness spectrum, therefore, is less susceptible to the large astrophysical uncertainties associated with high-z, low-mass groups.

Our model for the SZ profile from groups and clusters reproduces the pressure profile of massive X-ray clusters in the local universe. This provides significant constraints on the ICM models of the present-day massive clusters leaving only the redshift dependent parameters unknown. Adopting wide priors on these parameters, we determine astrophysical uncertainties in the skewness spectrum to be about $33 \%$. This is in contrast to the SZ power spectrum which is uncertain at the level of $50 \%$, primarily because a significant fraction of the power comes from high-z, low-mass groups with $M_{500} \lesssim 2 \times 10^{14} h^{-1} M_{\odot}$ and $z \gtrsim 0.6$. Such objects have not been studied in as much detail as their high mass counterparts, making their theoretical modeling and hence the SZ power spectrum highly uncertain. Other than the gas physics uncertainties, the theoretical modeling of the cluster mass function also add to the uncertainties in the bispectrum prediction. Because of the exponential nature of the mass function at the massive cluster end, a small change in cluster masses cause significant difference in the abundance of massive clusters (Bhattacharya et al. 2011).

The relation of the amplitude of the SZ skewness spectrum and the SZ power spectrum is robust to these astrophysical uncertainties. Indeed, the overall uncertainty of the relation is only $7 \%$ within the allowed range of ICM parameters. The skewness and the power spectrum amplitudes of the thermal SZ effect is related as $B_{\mathrm{SZ}}^{\Lambda}=A_{\mathrm{tSZ}}^{1.4}$; consequently, the skewness spectrum varies as $B_{\mathrm{SZ}}^{\Lambda} \propto \sigma_{8}^{11.6}$. Given the astrophysical uncertainty of $33 \%$, the skewness spectrum measurements have the potential to constrain $\sigma_{8}$ to better than $3 \%$ accuracy. Since the SZ skewness spectrum gets its contribution primarily from the thermal component, the SZ skewness spectrum measurements can constrain the thermal SZ component uniquely. We can then use the skewness spectrum measurements to break the degeneracy between thermal and the kinetic power spectrum measurements.

We show that the current high resolution experiments, such as ACT, Planck, and SPT, have the potential to measure the skewness spectrum. For example, using the full $2500 \mathrm{deg}^{2}$ of data, SPT should be able to detect the $\mathrm{SZ}$ skewness spectrum with a signal-to-noise $\approx 16$. Indeed, the SZ skewness signal we predict in this study is detected by the ACT collaboration at $5 \sigma$ (Wilson et al. 2012). We note however that our noise calculation does not account for all possible sources, as we make several simplified assumptions. For example, the contribution of the trispectrum is assumed to be negligible and we ignore any spatial correlation between the tSZ and CIB. However, as most of the SZ trispectrum signal comes from massive clusters (Shaw et al. 2009), masking out these objects should help reduce the SZ trispectrum contribution to the non-Gaussian component of the variance of the skewness spectrum. Also we assume the lensing 3pt function and its correlation with the SZ 3-pt function 
is zero, because CMB lensing dominates at $\ell \leq 2000$ while the SZ skewness spectrum gets most of the signal at $\ell \sim 4000$ (see Fig 8, right panel). In this study we also assume the kSZ bispectrum is negligible. While this assumption is valid for the linear part of the $\mathrm{kSZ}$ effect, the non-linear kSZ effect contributes a non-zero bispectrum which is not included in our calculation. However, given that the overall magnitude of the kSZ effect is about 20$40 \%$ of the thermal SZ amplitude, the non-linear kSZ bispectrum should be small. Nevertheless, in order to use the SZ skewness spectrum as a robust cosmological probe, these assumptions must be tested using mock catalogs. We will present such study in our future work.

We acknowledge useful discussions with John Carl- strom, Salman Habib, and Katrin Heitmann. We also thank the anonymous referee for useful suggestions. As this work neared completion, we learned about similar work by members of the ACT collaboration with whom we exchanged correspondence. This work was supported by the NSF grant AST-1009811. SB also acknowledges support by the NSF grant ANT-0638937 and by Argonne National Laboratory's resources under U.S. Department of Energy contract DE-AC02-06CH11357. DN acknowledges support by NASA ATP grant NNX11AE07G, NASA Chandra Theory grant GO213004B, Research Corporation, and by Yale University. TC is supported by the NSF grant ANT-0638937. GH is supported by NSERC and CIfAR and thanks KICP and FNAL for their hospitality.

\section{REFERENCES}

Arnaud, M., Pratt, G. W., Piffaretti, R., Böhringer, H., Croston, J. H., \& Pointecouteau, E. 2010, A\&A, 517, A92

Battaglia, N., Bond, J. R., Pfrommer, C., \& Sievers, J. L. 2012, ApJ, 758, 75

Battaglia, N., Bond, J. R., Pfrommer, C., Sievers, J. L., \& Sijacki, D. 2010, ApJ, 725, 91

Benson, B. A. et al. 2011, ArXiv e-prints, 1112.5435

Bhattacharya, S., Di Matteo, T., \& Kosowsky, A. 2008, MNRAS, 389,34

Bhattacharya, S., Habib, S., \& Heitmann, K. 2011a, ArXiv e-prints, 1112.5479

Bhattacharya, S., Heitmann, K., White, M., Lukić, Z., Wagner, C., \& Habib, S. 2011, ApJ, 732, 122

Bryan, G. L., \& Norman, M. L. 1998, ApJ, 495, 80

Chaudhuri, A., \& Majumdar, S. 2011, ApJ, 728, L41

Cooray, A. 2000, Phys. Rev. D, 62, 103506

Cooray, A., \& Hu, W. 2000, ApJ, 534, 533

Cooray, A., Hu, W., \& Tegmark, M. 2000, ApJ, 540, 1

Duffy, A. R., Schaye, J., Kay, S. T., \& Dalla Vecchia, C. 2008, MNRAS, 390, L64

Dunkley, J. et al. 2011, ApJ, 739, 52

Efstathiou, G., \& Migliaccio, M. 2011, ArXiv e-prints, 1106.3208

Fowler, J. W. et al. 2010, ApJ, 722, 1148

Giodini, S. et al. 2009, ApJ, 703, 982

Hu, W. 2000, Phys. Rev. D, 62, 043007

Jungman, G., Kamionkowski, M., Kosowsky, A., \& Spergel, D. N. 1996, Phys. Rev. D, 54, 1332

Kay, S. T., Thomas, P. A., Jenkins, A., \& Pearce, F. R. 2004, MNRAS, 355, 1091

Knox, L. 1995, Phys. Rev. D, 52, 4307

Komatsu, E., \& Seljak, U. 2002, MNRAS, 336, 1256

Komatsu, E. et al. 2011, ApJS, 192, 18

Komatsu, E., \& Spergel, D. N. 2001, Phys. Rev. D, 63, 063002

Lau, E. T., Kravtsov, A. V., \& Nagai, D. 2009, ApJ, 705, 1129

Lewis, A., Challinor, A., \& Lasenby, A. 2000, ApJ, 538, 473

Lueker, M. et al. 2010, ApJ, 719, 1045

Marriage, T. A. et al. 2011, ApJ, 731, 100

Mesinger, A., McQuinn, M., \& Spergel, D. N. 2012, MNRAS, 422, 1403

McCarthy, I. G. et al. 2010, MNRAS, 406, 822

Munshi, D., \& Heavens, A. 2010, MNRAS, 401, 2406

Nagamine, K., Ostriker, J. P., Fukugita, M., \& Cen, R. 2006, ApJ, 653, 881
Navarro, J. F., Frenk, C. S., \& White, S. D. M. 1996, ApJ, 462, 563

-.1997, ApJ, 490, 493

Negrello, M., Perrotta, F., González-Nuevo, J., Silva, L., de Zotti, G., Granato, G. L., Baccigalupi, C., \& Danese, L. 2007, MNRAS, 377, 1557

Nelson, K., Rudd, D. H., Shaw, L., \& Nagai, D. 2011, ArXiv e-prints, 1112.3659

Ostriker, J. P., Bode, P., \& Babul, A. 2005, ApJ, 634, 964

Pratt, G. W. et al. 2010, A\&A, 511, A85+

Puchwein, E., Sijacki, D., \& Springel, V. 2008, ApJ, 687, L53

Rasia, E., Tormen, G., \& Moscardini, L. 2004, MNRAS, 351, 237

Reichardt, C. L. et al. 2011, ArXiv e-prints, 1111.0932

Rubiño-Martín, J. A., \& Sunyaev, R. A. 2003, MNRAS, 344, 1155

Shaw, L. D., Nagai, D., Bhattacharya, S., \& Lau, E. T. 2010, ApJ, 725, 1452

Shaw, L. D., Rudd, D. H., \& Nagai, D. 2011, ArXiv e-prints, 1109.0553

Shaw, L. D., Zahn, O., Holder, G. P., \& Doré, O. 2009, ApJ, 702, 368

Shirokoff, E. et al. 2011, ApJ, 736, 61

Short, C. J., Thomas, P. A., \& Young, O. E. 2012, ArXiv e-prints, 1201.1104

Sijacki, D., Springel, V., di Matteo, T., \& Hernquist, L. 2007, MNRAS, 380, 877

Sunyaev, R. A., \& Zeldovich, I. B. 1980, ARA\&A, 18, 537

Teyssier, R., Moore, B., Martizzi, D., Dubois, Y., \& Mayer, L. 2011, MNRAS, 414, 195

Tinker, J., Kravtsov, A. V., Klypin, A., Abazajian, K., Warren,

M., Yepes, G., Gottlöber, S., \& Holz, D. E. 2008, ApJ, 688, 709

Trac, H., Bode, P., \& Ostriker, J. P. 2011, ApJ, 727, 94

Vanderlinde, K. et al. 2010, ApJ, 722, 1180

Vieira, J. D. et al. 2010, ApJ, 719, 763

Vikhlinin, A., Kravtsov, A., Forman, W., Jones, C., Markevitch, M., Murray, S. S., \& Van Speybroeck, L. 2006, ApJ, 640, 691

Wilson, M. J., Sherwin, B. D., Hill, J. C., et al. 2012, arXiv:1203.6633

Zahn, O. et al. 2011, ArXiv e-prints, 1111.6386 